\title{
Analysis of the association between KIN17 expression and the clinical features/prognosis of epithelial ovarian cancer, and the effects of KIN17 in SKOV3 cells
}

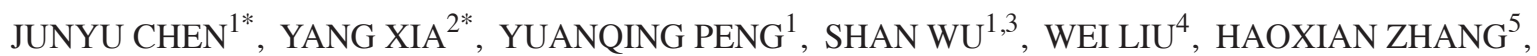 \\ TIAN WANG ${ }^{1}$, ZHAOYUN YANG $^{6}$, SHUHUA ZHAO ${ }^{1}$ and LIJING ZHAO ${ }^{6}$
}

Departments of ${ }^{1}$ Gynecology and Obstetrics, and ${ }^{2}$ Pathology, The Second Hospital of Jilin University,

Changchun, Jilin 130022; ${ }^{3}$ Department of Reproductive Endocrinology, Women's Hospital, Zhejiang University,

School of Medicine, Hangzhou, Zhejiang 310006; ${ }^{4}$ Research Center for Circular Economy and Pollution Prevention, Jilin

Academy of Environmental Sciences, Changchun, Jilin 130021; ${ }^{5}$ Department of Pharmacy, Xuchang Municipal Hospital,

Xuchang, Henan 461000; ${ }^{6}$ Department of Recovery, Nursing School of Jilin University, Changchun, Jilin 130012, P.R. China

Received October 4, 2020; Accepted February 19, 2021

DOI: 10.3892/ol.2021.12736

\begin{abstract}
DNA double-strand breaks (DSBs) are an important mechanism of chemotherapy in epithelial ovarian cancer (EOC). Kin17 DNA and RNA binding protein (KIN17) serves a crucial role in DSB repair. In the present study, the association between KIN17 and EOC, and the effects of KIN17 on EOC cells in vitro were evaluated. A bioinformatics method was used to determine the mRNA expression levels of KIN17 in EOC and its association with EOC prognosis including overall survival (OS) and progression free survival (PFS) time. Western blotting and immunohistochemical staining were used to evaluate the expression levels of KIN17 in EOC samples. Kaplan-Meier and Cox regression analyses were utilized to analyze risk factors for the OS of patients with EOC. A Cell Counting Kit-8 assay was performed to explore the roles of KIN17 in SKOV3 cells. Both the transcription and expression of KIN17 were upregulated in EOC tissues. Furthermore, the OS of patients with EOC with high mRNA expression levels of KIN17 was shorter than that of patients with EOC with low expression levels. High KIN17 expression was an independent risk factor for EOC prognosis. Furthermore, KIN17 knockdown inhibited the proliferation of SKOV3 cells, enhanced the sensitivity of the cells to cisplatin and inhibited the migration ability of the cells. These results suggested that KIN17 may act as an ideal candidate for therapy
\end{abstract}

Correspondence to: Dr Shuhua Zhao, Department of Gynecology and Obstetrics, The Second Hospital of Jilin University, 218 Ziqiang Street, Changchun, Jilin 130022, P.R. China

E-mail: zhaoshuhua-1966@163.com

*Contributed equally

Key words: Kin17 DNA and RNA binding protein, epithelial ovarian cancer, DNA double-strand break repair, bioinformatics analysis, prognosis and as a prognostic biomarker of EOC, although the underlying mechanisms require further exploration.

\section{Introduction}

Epithelial ovarian cancer (EOC) is the most lethal gynecologic malignancy worldwide, with a 5-year survival rate of $46.5 \%$ between 2005 to 2011 (1), as well as 230,000 new cases and 150,000 deaths annually in 2012 (2). Due to the increasing research on traditional and novel targeted drugs, the lives of patients with EOC are being prolonged. Researchers have revealed that after treatment with surgery and platinum-based chemotherapy, $50 \%$ of patients with EOC with stage III disease could live for $>5$ years (3). At present, the median survival of recurrent patients sensitive to platinum is 3 years; however, for patients with EOC who are resistant to platinum it is only 1 year (4). The remission rate of patients with EOC has increased markedly with the combination of platinum-based traditional chemotherapy and targeting therapies, including VEGF inhibitors and poly(ADP-ribose) polymerase 1 inhibitors $(3,5)$. However, these two classes of drug are only used for patients who are sensitive to platinum (6). Therefore, exploring novel efficient targeted drugs that can enhance the sensitivity of patients with EOC to platinum is of significance to improve patient prognosis.

DNA double-strand breaks (DSBs) are a key mechanism of platinum-based chemotherapy in EOC. When entering EOC cells, platinum binds to DNA spontaneously to induce platinum-DNA cross-links that damage the normal structure and function of DNA (7). As a result, cell cycle progression is blocked and cell death is induced $(8,9)$. DNA damage repair could be induced by excessive DNA DSB aggregation in EOC cells, which causes cells to be resistant to platinum (9). The sensitivity of EOC cells to platinum depends on their ability to induce DNA damage repair spontaneously (5). DNA DSBs are mainly repaired by homologous recombination signaling pathways (10). Previous research has demonstrated that homologous recombination defects are obvious in patients with ovarian cancer with BRCA1 gene mutations, resulting 
in higher sensitivity to chemotherapy than in patients without BRCA1 gene mutations (11). It is estimated that $~ 50 \%$ of high-grade serous adenocarcinomas feature homologous recombination $(3,12)$. Therefore, identifying molecules that can inhibit homologous recombination in EOC cells is key to enhancing the sensitivity of patients with EOC to platinum.

Kin17 DNA and RNA binding protein (KIN17), a DNA- and RNA-binding protein that is extremely well conserved across biological evolution, is considered critical to the proliferation and survival of mammalian cells, including normal and cancerous cells (13). Importantly, the KIN17 protein has been demonstrated to be involved in the DNA damage repair process by regulating the homologous recombination signaling pathway $(14,15)$. When the KIN17 gene is silenced, DNA replication, DNA damage repair, cell cycle progression and proliferation are inhibited in breast cancer cells; however, sensitivity to chemotherapy is increased (16). Proliferation, invasion and metastasis are inhibited by downregulation of the KIN17 protein, thereby inducing apoptosis and cell cycle arrest in cervical cancer cells (17). The KIN17 protein can induce the proliferation of liver cancer cells (18), and its upregulation is associated with the invasion and metastasis of non-small cell lung cancer (19). However, to the best of our knowledge, the effect of KIN17 in ovarian cancer remains unclear and requires further exploration.

The aim of the present study was to investigate the possible association between KIN17 expression and the clinical/pathological features, as well as the overall survival (OS) of patients with EOC, and to verify the effects of KIN17 on EOC in vitro in order to provide a theoretical basis for future research on novel targeted drugs.

\section{Materials and methods}

Bioinformatics analysis. The Oncomine database (https://www.oncomine.org/resource/login.html) was used to analyze the difference in KIN17 gene expression between EOC and normal ovarian tissues [datasets from The Cancer Genome Atlas (TCGA) Ovarian (Affymetrix ID: 205664_at), Lu Ovarian (Affymetrix ID: 37778_at) (20) and TCGA Ovarian 2 (Affymetrix ID: 0-007853661)], as well as between borderline ovarian surface epithelial-stromal tumor and ovarian cancer tissues [dataset from Anglesio Ovarian (GSE12172, Affymetrix ID: 205664_at)] (21). Furthermore, the association between KIN17 mRNA expression and the sensitivity of EOC cells to platinum was examined using the Oncomine database [dataset derived from Gyorffy CellLine (GSE11812, Affymetrix ID: 205664_at)] (22). The Kaplan-Meier database (http://kmplot.com/analysis/index. $\mathrm{php}$ ? $\mathrm{p}=$ service $\&$ cancer $=$ ovar) was used to examine the association between the transcriptional levels of KIN17 and the prognoses of patients with ovarian cancer. All the data in this part were analyzed from 15 datasets (23). The follow-up time was between 2 and 240 months. The expression range of the probe in 205664_at was between 4 and 1,845; 533 and 377 were selected as the cut-off values for OS and progression-free survival (PFS) analysis, respectively. The expression range of the probe in 236887_at was between 6 and 558; 127 was selected as the cut-off value for OS analysis. All cut-off values between the lower and upper quartiles were computed, and the best performing threshold was used as the cut-off.
Specimens. The present study was approved by the Research Ethics Committee of The Second Hospital of Jilin University (Changchun, China). All patients were informed and agreed to participate in the present study. Paraffin-embedded specimens were collected at The Second Hospital of Jilin University (Changchun, China) from 56 patients with EOC and five patients with mucous cystadenoma who were diagnosed between January 2012 and January 2018. The median age of the patients was 52 years (range, 32-76 years). All patients were female. The inclusion criteria were as follows: i) Initially diagnosed with EOC and treated with early comprehensive staging laparotomy or ideal cytoreductive surgery; ii) the EOC diagnosis was confirmed by the Pathology Department of The Second Hospital of Jilin University; iii) postoperative pathology results were measured strictly by Federation International of Gynecology and Obstetrics (FIGO) staging criteria (24); iv) standardized platinum-based chemotherapy was administered postoperatively; and v) complete follow-up data were available. The exclusion criteria were as follows: i) Individual history of other malignant tumors; ii preoperative chemo/radiotherapy; and iii) secondary ovarian cancer. All patient blood samples were collected before surgery and immediately analyzed for the levels of CA125 and human epididymis protein 4 (HE4). Fresh frozen $\left(-80^{\circ} \mathrm{C}\right)$ specimens, including cancer tissues and adjacent tissues, were collected from 20 patients with EOC who were diagnosed at the same hospital between February 2018 and August 2019. All patients with EOC received standard platinum-based chemotherapy following cytoreductive surgeries. As shown in Table I, available clinical/pathological data were extracted from the Medical Record Database of The Second Hospital of Jilin University. All 56 patients with EOC were followed up, and the OS of these patients was calculated. The median follow-up for patients with EOC was 31 months, ranging between 7 and 77 months.

Immunohistochemistry. Immunohistochemical (IHC) staining was performed as described previously (25). Briefly, after being fixed in $10 \%$ formalin at room temperature for $24 \mathrm{~h}$, EOC tissues were embedded in paraffin and cut into $3-\mu \mathrm{m}$ thick sections. After being deparaffinized by xylol and rehydrated by descending alcohol series (100, 85 and 70\% alcohol, $10 \mathrm{~min}$ for each concentration) at room temperature, the sections were soaked in EDTA retrieval buffers (cat. no. AR0023; Wuhan Boster Biological Technology, Ltd.) and heated in a microwave oven at $98^{\circ} \mathrm{C}$ for $15 \mathrm{~min}$. Then the sections were cooled naturally at room temperature. Non-specific binding was blocked using 5\% bovine serum albumin (BSA; cat. no. AR1006; Wuhan Boster Biological Technology, Ltd.) at room temperature for $20 \mathrm{~min}$. Subsequently, the histological sections were stained with rabbit anti-KIN17 antibody (dilution, 1:100; cat. no. PB0639; Wuhan Boster Biological Technology, Ltd.) at $4^{\circ} \mathrm{C}$ overnight. Goat anti-rabbit IgG conjugated with horseradish peroxidase (dilution, 1:200; cat. no. S0001; Affinity Biosciences) was used as the secondary antibody, and this staining procedure was carried out at $37^{\circ} \mathrm{C}$ for $30 \mathrm{~min}$. Reactive products were visualized with 3,3'-diaminobenzidene (cat. no. AR1022; Wuhan Boster Biological Technology, Ltd.) as the chromogen, and the sections were counterstained with hematoxylin $(0.1 \%$; cat. no. AR0005; Wuhan Boster Biological Technology, Ltd.) at room temperature for $2 \mathrm{~min}$. Histological images were captured under a light microscope (BX51; Olympus Corporation) with 
Table I. Association between KIN17 protein expression and clinical features of patients with epithelial ovarian cancer.

\begin{tabular}{|c|c|c|c|c|c|}
\hline \multirow[b]{2}{*}{ Clinical features } & \multirow[b]{2}{*}{ No. of cases (\%) } & \multicolumn{2}{|c|}{ KIN17 expression } & \multirow[b]{2}{*}{$\chi^{2}$} & \multirow[b]{2}{*}{ P-value } \\
\hline & & High expression, $\mathrm{n}$ & Low expression, $\mathrm{n}$ & & \\
\hline \multicolumn{6}{|l|}{ Age, years } \\
\hline$<52$ & $27(48.21)$ & 16 & 11 & & \\
\hline$\geq 52$ & $29(51.79)$ & 16 & 13 & 0.095 & 0.757 \\
\hline \multicolumn{6}{|l|}{ FIGO stage } \\
\hline $\mathrm{I}+\mathrm{II}$ & $23(41.07)$ & 10 & 13 & & \\
\hline III+IV & $33(58.93)$ & 22 & 11 & 2.976 & 0.085 \\
\hline \multicolumn{6}{|l|}{ Tumor size, cm } \\
\hline$\leq 8$ & $28(50.00)$ & 14 & 14 & & \\
\hline$>8$ & $28(50.00)$ & 18 & 10 & 1.167 & 0.280 \\
\hline \multicolumn{6}{|l|}{ EOC type $^{\mathrm{a}}$} \\
\hline Type I & $26(46.43)$ & 14 & 12 & & \\
\hline Type II & $30(53.57)$ & 18 & 12 & 0.215 & 0.643 \\
\hline \multicolumn{6}{|l|}{ Pathological grade } \\
\hline Low level & $23(41.07)$ & 13 & 10 & & \\
\hline High level & $33(58.93)$ & 19 & 14 & 0.006 & 0.938 \\
\hline \multicolumn{6}{|c|}{ Ascites volume, ml } \\
\hline$<500$ & $31(55.36)$ & 16 & 15 & & \\
\hline$\geq 500$ & $25(44.64)$ & 16 & 9 & 0.867 & 0.352 \\
\hline \multicolumn{6}{|c|}{ Lymphatic metastasis $^{c}$} \\
\hline Positive & $17(37.78)$ & 13 & 4 & & \\
\hline Negative & $28(62.22)$ & 12 & 16 & 4.840 & $0.028^{b}$ \\
\hline \multicolumn{6}{|l|}{$\mathrm{CA} 125, \mathrm{U} / \mathrm{ml}^{\mathrm{d}}$} \\
\hline$<500$ & $25(47.17)$ & 17 & 8 & & \\
\hline$\geq 500$ & $28(52.83)$ & 15 & 13 & 1.149 & 0.284 \\
\hline \multicolumn{6}{|l|}{$\mathrm{HE} 4, \mathrm{pmol} / \mathrm{l}^{\mathrm{d}}$} \\
\hline$<500$ & $26(68.42)$ & 17 & 9 & & \\
\hline$\geq 500$ & $12(31.58)$ & 6 & 6 & 0.813 & 0.367 \\
\hline
\end{tabular}

${ }^{a}$ Type I, low-grade serous carcinoma, low-grade endometrioid carcinoma, mucinous carcinoma, clear cell carcinoma and malignant Brenner tumor; Type II, high-grade serous carcinoma, high-grade endometrioid carcinoma, carcinosarcoma and undifferentiated carcinoma. ${ }^{b} \mathrm{P}<0.05$. FIGO, Federation International of Gynecology and Obstetrics; HE4, human epididymis protein 4; KIN17, Kin17 DNA and RNA binding protein. ${ }^{\mathrm{C}}$ Data incomplete as some patients could not tolerate the comprehensive staging laparotomy surgery, so lymph node excision was not performed. ${ }^{\mathrm{d}}$ Data incomplete as some medical record data were lost.

an objective magnification of $\mathrm{x} 200$ or $\mathrm{x} 400$. The intensity was scored as follows: 0 , no color; 1 , light yellow; 2 , yellow brown; and 3 , brown. The proportion of positive tumor cells was scored as: $0,0-4 ; 1,5-24 ; 2,25-49 ; 3,50-74$; and $4,75-100 \%$. The overall score of each sample was obtained by multiplying the two scores. A score of $>4$ was defined as high KIN17 expression, and a score of $\leq 4$ was defined as low KIN17 expression. The IHC staining was double-blinded and scored independently by two experienced pathologists working at The Pathology Department of the Second Hospital of Jilin University.

Western blot analysis. Protein samples from fresh frozen tissues or stably transfected cells were extracted using cold RIPA lysis buffer (cat. no. P0013K; Beyotime Institute of Biotechnology). Subsequently, the protein samples were quantified according to the Bradford method using an Easy Protein Quantitative Kit (cat. no. DQ101-01; TransGen
Biotech Co., Ltd.). A total of $30 \mu \mathrm{g}$ protein sample was added to each lane, resolved by $12 \%$ SDS-PAGE, separated electrophoretically and transferred onto polyvinylidene fluoride membranes (EMD Millipore). The non-specific binding sites of the membrane were blocked with $5 \%$ dried skim milk at $37^{\circ} \mathrm{C}$ for $1 \mathrm{~h}$. Subsequently, the membrane was incubated with primary antibodies, including rabbit anti-KIN17 (dilution, 1:2,000; cat. no. PB0639; Wuhan Boster Biological Technology, Ltd.) and mouse anti- $\beta$-actin (dilution, 1:1,500; cat. no. HC201-01; TransGen Biotech Co., Ltd.) at $4{ }^{\circ} \mathrm{C}$ overnight, followed by incubation with horseradish peroxidase-tagged goat anti rabbit IgG (dilution, 1:4,000; cat. no. S0001) and goat anti mouse IgG (dilution, 1:4,000; cat. no. S0002) (both Affinity Biosciences) at room temperature for $2 \mathrm{~h}$, respectively. Finally, the protein expression levels were detected with an electrochemiluminescence plus kit (cat. no. KF001; Affinity Biosciences), and the densities of 
the specific bands were semi-quantified using an imaging densitometer (Clinx Science Instruments Co., Ltd.) and the software Gel Analysis (Clinx Science Instruments Co., Ltd.) that is provided with the densitometer.

Cell culture and stably transfected cell line development. The human EOC cell line SKOV3 was originally obtained from The Basic Medical College of Jilin University (Changchun, China). The cells were cultured in Iscove's modified Dulbecco's medium (IMDM; HyClone; Cytiva) supplemented with $10 \%$ fetal bovine serum (Gibco; Thermo Fisher Scientific, Inc.) and $1 \%$ penicillin-streptomycin (Invitrogen; Thermo Fisher Scientific, Inc.) at $37^{\circ} \mathrm{C}$ in a humidified atmosphere with $5 \% \mathrm{CO}_{2}$.

The construction of short hairpin RNA (shRNA)-KIN17 (NM_012311.3) was performed by GeneCopoeia, Inc. The stably transfected SKOV3 cell line was transfected with shRNA-KIN17 (cat. no.CS-RSH086442-CU6-01; GeneCopoeia,Inc.) based with the plasmid psi-U6 (GeneCopoeia, Inc.) using Lipofectamine 3000 reagent (Invitrogen; Thermo Fisher Scientific, Inc.) according to the manufacturer's protocols. SKOV3 cells were seeded into a six-well plate at a density of $8 \times 10^{5}$ cells/well. Then, $2.5 \mu \mathrm{g}$ shRNA-KIN17 or shRNA-Scramble (treatment groups) and only Lipofectamine 3000 reagent (negative control) were added into the wells of different groups. Following incubation at $37^{\circ} \mathrm{C}$ for $96 \mathrm{~h}$, the transfected cells were selected with $5 \mu \mathrm{g} / \mathrm{ml}$ puromycin (cat. no. P1033; Biotopped Life Sciences) for 2 weeks before experimentation, and the surviving cells were continuously cultured as a stably transfected cell line. The transfection efficiency was detected by fluorescence microscopy, reverse transcription-quantitative PCR (RT-qPCR) and western blotting.

$R T$ - $q P C R$. RNA was extracted from the stably transfected cells using TRIzol reagent (Invitrogen; Thermo Fisher Scientific, Inc.), followed by quantification with a NanoDrop 2000 instrument (NanoDrop Technologies; Thermo Fisher Scientific, Inc.). Following reverse transcription with a cDNA synthesis kit (Beijing Transgen Biotech Co., Ltd.), cDNA was used for PCR amplification using a SYBR Green real-time PCR kit (Beijing Transgen Biotech Co., Ltd.) using an ABI-Q3 system (Thermo Fisher Scientific, Inc.). The primers used were as follows: KIN17 Gene forward, 5'-GGACCCAGAAACTAT CCGCC-3' and reverse, 5'-TTCCCTTCCAGGCCTCTTCT-3'; and GAPDH forward, 5'-GAAGGTGAAGGTCGGAGTC-3' and reverse, 5'-GAAGATGGTGATGGGATTTC-3'. GAPDH was used as an internal control gene. Reverse transcription was performed with Anchored Oligo(dT) at $42^{\circ} \mathrm{C}$ for $15 \mathrm{~min}$, followed by incubating with Random Primer (N9) at $25^{\circ} \mathrm{C}$ for $10 \mathrm{~min}, 42^{\circ} \mathrm{C}$ for $15 \mathrm{~min}$ and $85^{\circ} \mathrm{C}$ for $5 \mathrm{sec}$ using a 96-well Thermal Cycler (Thermo Fisher Scientific, Inc.). PCR was performed at $94^{\circ} \mathrm{C}$ for $30 \mathrm{sec}$, followed by 45 cycles of amplification at $94^{\circ} \mathrm{C}$ for $5 \mathrm{sec}, 51^{\circ} \mathrm{C}$ for $15 \mathrm{sec}$ and $72^{\circ} \mathrm{C}$ for $10 \mathrm{sec}$ using an ABI-Q3 system (Thermo Fisher Scientific, Inc.). Relative mRNA expression levels were calculated using the $2^{-\Delta \Delta C q}$ method (26).

Cell counting kit-8 (CCK-8) assay. After being transfected with shRNA-KIN17 or shRNA Scramble, these SKOV3 cells and untreated SKOV3 cells were seeded into 96-well plates (1,000 cells/well). After 24, 48, 72, 96 and 120 h, CCK-8 reagent (10 $\mu \mathrm{l} /$ well; BIOSS) was added to each well. Following culture at $37^{\circ} \mathrm{C}$ for $2 \mathrm{~h}$, the absorbance of each well was measured at $450 \mathrm{~nm}$ using a microplate reader (Cytation 5; BioTek Instruments, Inc.). The stably transfected SKOV3 cells were seeded into 96-well plates at a density of $5 \times 10^{3}$ cells/well and incubated at $37^{\circ} \mathrm{C}$ overnight. After exposure to cisplatin at $0,0.5$, $1,2,4,8$ or $16 \mu \mathrm{g} / \mathrm{ml}$ at $37^{\circ} \mathrm{C}$ for $48 \mathrm{~h}, \mathrm{CCK}-8$ reagent $(10 \mu \mathrm{l} / \mathrm{well}$; BIOSS) was used to detect the proliferation rates of SKOV3 cells and sensitivity of SKOV3 cells to cisplatin at $37^{\circ} \mathrm{C}$ for $2 \mathrm{~h}$.

Transwell assay. For the migration assay, following $12 \mathrm{~h}$ of serum starvation treatment, untreated SKOV3, shRNA-KIN17transfected SKOV3 and shRNA-Scramble-transfected SKOV3 cells were adjusted to a density of $3 \times 10^{5}$ cells $/ \mathrm{ml}$ using serum-free Iscove's modified Dulbecco's medium (IMDM; containing or not containing cisplatin at $1 \mu \mathrm{g} / \mathrm{ml})$. Subsequently, $100 \mu \mathrm{l}$ single cell suspension was added to the apical chamber ( $8-\mu$ m polycarbonate membrane; Corning, Inc.), and $600 \mu 1$ IMDM with $10 \%$ FBS (containing or not containing cisplatin at $1 \mu \mathrm{g} / \mathrm{ml}$ ) was added to the bottom chamber. The cells were allowed to migrate through the polycarbonate membrane for $40 \mathrm{~h}$ at $37^{\circ} \mathrm{C}$ with $5 \% \mathrm{CO}_{2}$. Subsequently, the cells remaining on the upper surface were wiped off with a cotton ball. The migration cells were fixed with 4\% paraformaldehyde at room temperature for $30 \mathrm{~min}$ and stained with $0.1 \%$ crystal violet (cat. no. C6470; Biotopped Life Sciences) at room temperature for $10 \mathrm{~min}$, and then observed using an optical light microscope (magnification, x200). Subsequently, the cells were observed and quantified in five random fields of view (magnification, x200). Mean integrated optical density (IOD) was measured using Image-Pro Plus 6.0 software (Media Cybernetics, Inc). The IOD fold changes of the treatment groups were also analyzed. All experiments were performed in triplicate.

Statistical analysis. Continuous data are presented as mean and \pm standard deviation. The association between the expression levels of KIN17 and the clinical/pathological features of patients with EOC was analyzed using the $\chi^{2}$ test. Student's two-tailed t-test was used to verify significant differences in KIN17 expression between EOC and EOC-adjacent tissues. In addition, one-way ANOVA and Tukey's test were used for multiple comparisons. OS curves were generated using the Kaplan-Meier method and compared using a log-rank test. Univariate analyses were performed using Kaplan-Meier. Multivariate analyses were performed using Cox regression models. All experiments were repeated three times independently. All data were analyzed using SPSS software (version 23.0; IBM Corp.). $\mathrm{P}<0.05$ was considered to indicate a statistically significant difference.

\section{Results}

Association between KINI7 DNA/mRNA levels and EOC. Using the Oncomine database, the association between the gene expression of KIN17 and EOC was analyzed. As shown in Fig. 1A, compared with that in normal ovary tissues, the transcription of KIN17 was upregulated in ovarian serous adenocarcinoma tissues $(\mathrm{P}<0.05)$, which was also demonstrated in Fig. 1B $(\mathrm{P}<0.05)$. Nevertheless, there was no significant difference in KIN17 mRNA expression between normal ovary tissues and other EOC tissues, including ovarian mucinous, endometrioid and clear cell adenocarcinoma tissues (Fig. 1B). Fig. 1C shows that the KIN17 DNA levels were upregulated in ovarian serous adenocarcinoma 
A

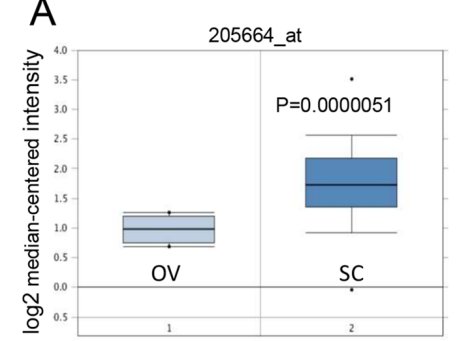

D 205664_at

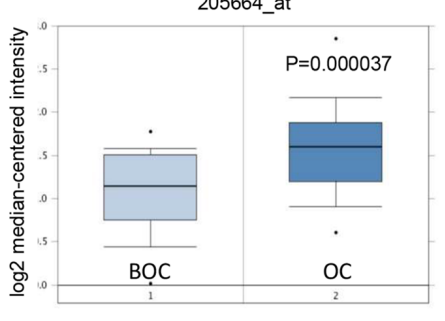

G

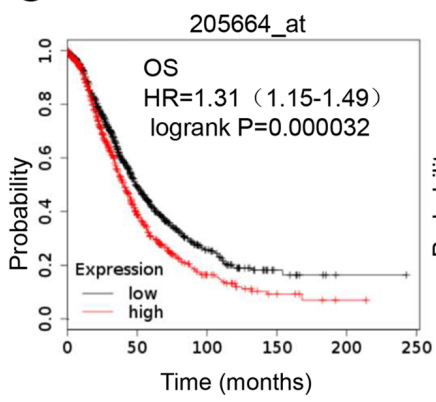

$\mathrm{J}$
B
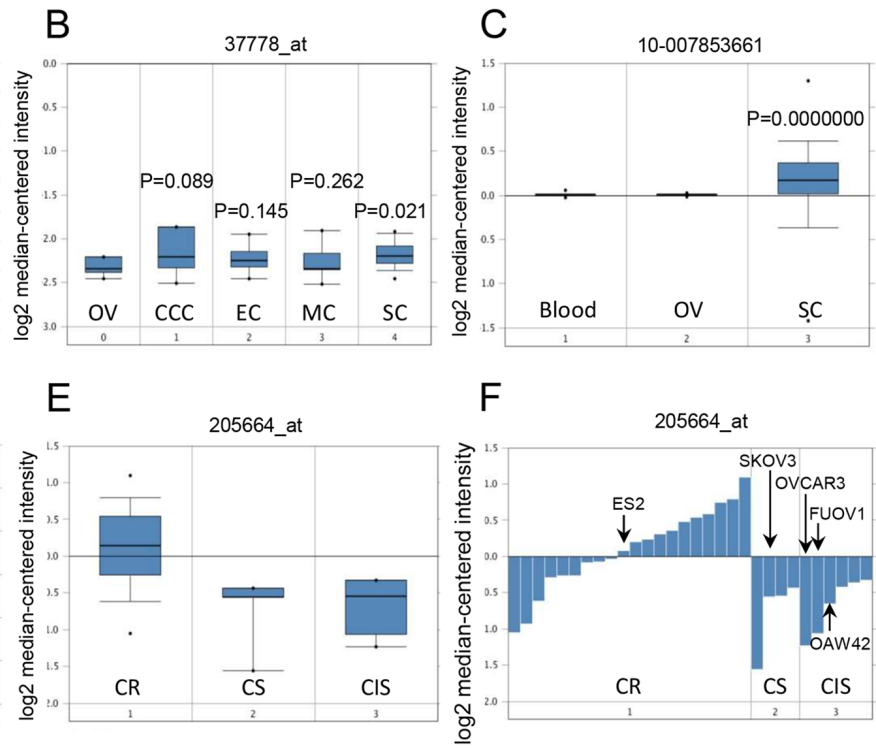

F 205664_at

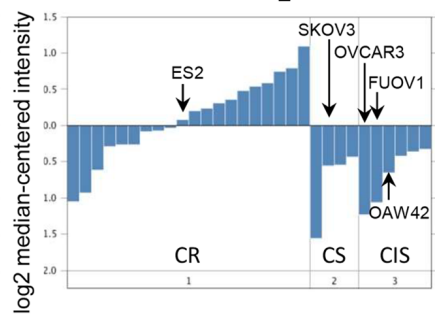

$\mathrm{H}$

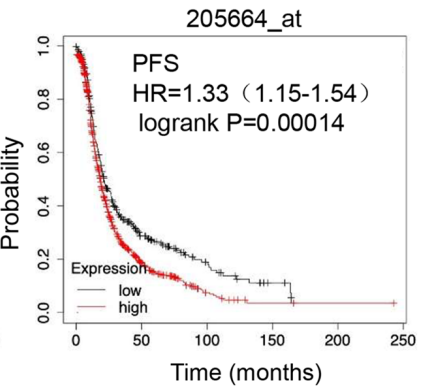

I

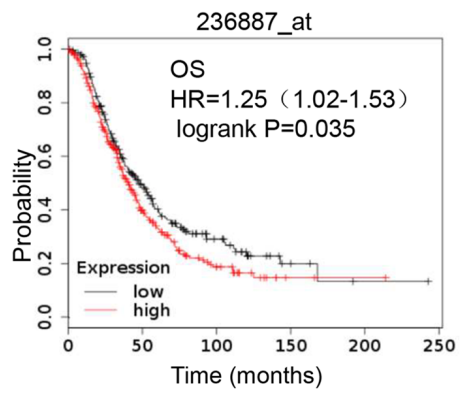

\begin{tabular}{|c|c|c|c|c|c|c|}
\hline \multirow{2}{*}{ KIN17 mRNA } & \multicolumn{4}{|c|}{ 205664_at } & \multicolumn{2}{|c|}{ 236887_at } \\
\hline & $\mathrm{N}$ & $\begin{array}{c}\text { Median OS } \\
\text { (months) }\end{array}$ & $\mathrm{N}$ & $\begin{array}{c}\text { Median PFS } \\
\text { (months) }\end{array}$ & $\mathrm{N}$ & $\begin{array}{c}\text { Median OS } \\
\text { (months) }\end{array}$ \\
\hline Low level & 925 & 49 & 345 & 21.13 & 294 & 48 \\
\hline High level & 731 & 40 & 914 & 18.27 & 361 & 40.54 \\
\hline
\end{tabular}

Figure 1. Bioinformatics analysis of the role of KIN17 gene expression in the development of OC and prognosis of patients with OC. (A) mRNA expression levels of KIN17 in OV and SC tissues (Affymetrix ID: 205664_at; 1. OV, n=8; 2. SC, n=586). (B) mRNA expression levels of KIN17 in OV and EOC tissues (Affymetrix ID: 37778_at; 0. OV, n=5; 1. CCC, n=7; 2. EC, $\mathrm{n}=9 ; 3$. MC, n=9; 4. SC, n=20). (C) DNA expression levels of KIN17 in blood, OV and SC tissues (Affymetrix ID: 10-007853661; 1. Blood, $n=431 ; 2$. OV, n=130; 3. SC, n=607). (D) mRNA expression levels of KIN17 in BOC and OC tissues (Affymetrix ID: 205664_at; 1. BOC, n=30; 2. OC, n=44). (E) mRNA expression levels of KIN17 in Gyorffy cell lines (Affymetrix ID: 205664_at; 1. CR, n=20; 2. CS, n=6; 3. CIS, $n=4)$. (F) mRNA expression levels of KIN17 in different EOC cell lines (Affymetrix ID: 205664_at; 1. CR; 2. CS; 3. CIS). (G) Association between KIN17 mRNA expression and the OS of patients with OC (Affymetrix ID: 205664_at). (H) Association between KIN17 mRNA expression and the PFS of patients with OC (Affymetrix ID: 205664_at). (I) Association between KIN17 mRNA expression and the OS of patients with OC (Affymetrix ID: 236887_at). (J) Sample numbers and median survival (OS or PFS) of patients with OC in different datasets. BOC, borderline ovarian surface epithelial-stromal tumor; CCC, clear cell adenocarcinoma; CIS, cisplatin intermediate sensitivity; CR, cisplatin resistant; CS, cisplatin sensitivity; EC, endometrioid adenocarcinoma; EOC, epithelial ovarian cancer; HR, hazard ratio; KIN17, Kin17 DNA and RNA binding protein; MC, mucinous adenocarcinoma; OC, ovarian cancer; OS, overall survival; OV, normal ovary; PFS, progression-free survival; SC, ovarian serous adenocarcinoma.

tissues compared with ovarian tissues $(\mathrm{P}<0.05)$. Furthermore, the transcription of KIN17 was higher in ovarian cancer tissues than borderline ovarian surface epithelial-stromal tumor tissues $(\mathrm{P}<0.05$; Fig. 1D). Gyorffy Cell Line Statistics obtained and analyzed from the Oncomine database revealed that cisplatin-resistant cancer cell lines exhibited high mRNA expression levels of KIN17 compared with cisplatin-sensitive and intermediate sensitive cancer cell lines (Fig. 1E). Additionally, KIN17 mRNA expression was identified to be increased in the ES-2 cell line, a cisplatin-resistant EOC cell line with a KIN17 expression value of 0.08 , compared with the OVCAR-3 cells with an expression value of -1.24 , FU-OV-1 cells with an expression value of -1.07 and OAW42 cells with an expression value of -0.66 (cisplatin sensitivity), as well as the SKOV3 cell line with an expression value of -0.56 (cisplatin intermediate sensitivity; Fig. 1F).

Association between KIN17 mRNA expression and the prognosis of patients with EOC. Using the Kaplan-Meier 

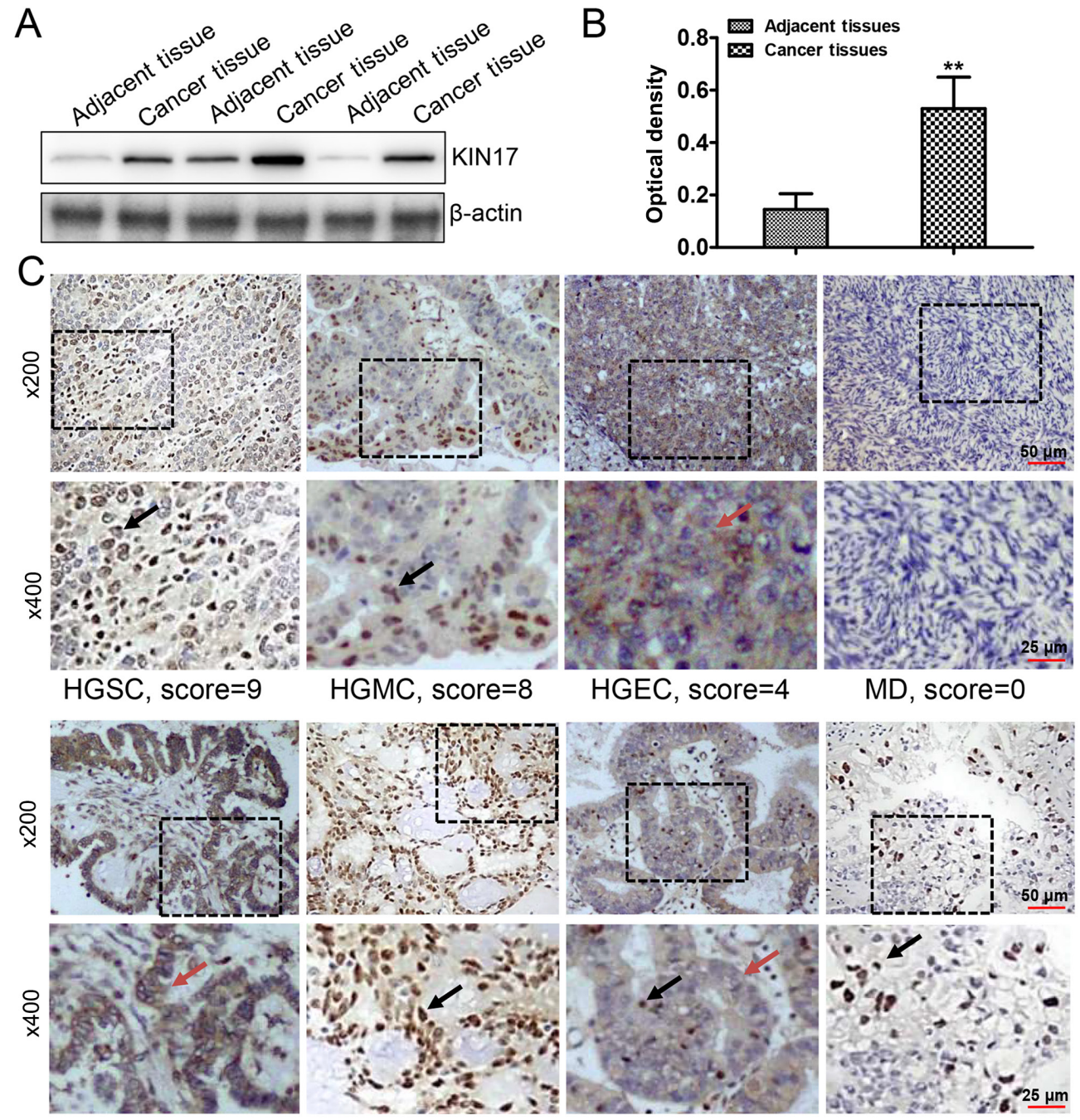

LGSC, score $=8$

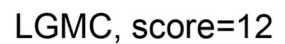

LGEC, score $=8$

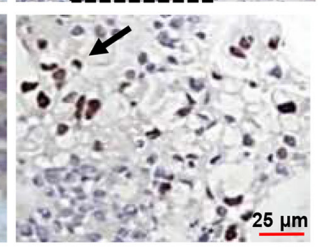

CCC, score $=4$

D

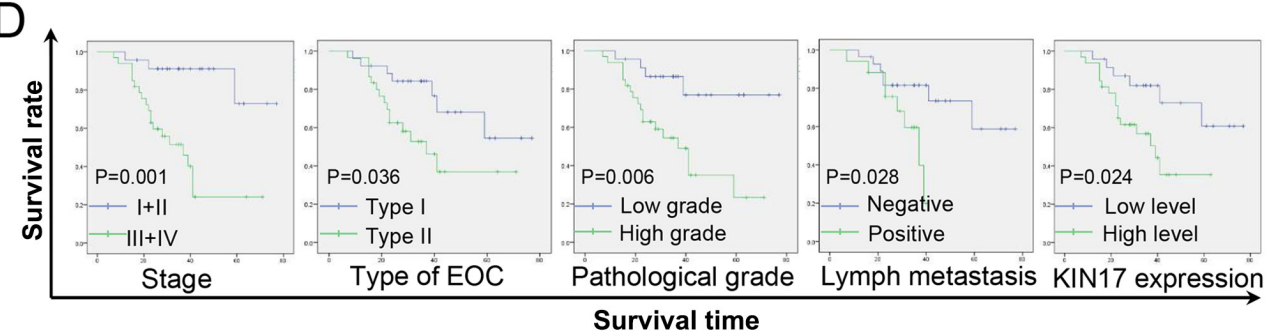

Figure 2. Analysis of the roles of KIN17 protein in the development and predictive prognosis of patients with EOC using IHC staining and western blotting. (A) Western blot of KIN17 in EOC and adjacent tissues. (B) Analysis of KIN17 expression level in EOC and adjacent tissues. (C) Typical IHC staining images for HGSC, HGMC, HGEC, MD, LGSC, LGMC, LGEC and CCC (magnification, x200 or x400). MD was used as a negative control. KIN17 protein expression in the cytoplasm was indicated using red arrows and KIN17 protein expression in the nucleus was indicated using black arrows. (D) Overall survival of patients with EOC according to Federation International of Gynecology and Obstetrics stages, EOC types, pathological grades, lymphatic metastasis and KIN17 expression. ${ }^{* *} \mathrm{P}<0.01$ vs. adjacent tissue group. CCC, clear cell carcinoma; EOC, epithelial ovarian cancer; HGEC, high-grade endometrioid carcinoma; HGMC, high-grade mucous carcinoma; HGSC, high-grade serous carcinoma; IHC, immunohistochemical; KIN17, Kin17 DNA and RNA binding protein; LGEC, low-grade endometrioid carcinoma; LGMC, low-grade mucous carcinoma; LGSC, low-grade serous carcinoma; MD, mucous cystadenoma.

database, the association between the transcription of KIN17 and EOC prognosis was investigated. As shown in Fig. 1G, I and J, compared with patients with EOC with low mRNA expression levels of KIN17, patients with EOC with high expression levels of KIN17 had a poorer OS (median, 49 months vs. 40 months in 205664_at, $\mathrm{P}<0.01$; median, 48 months vs. 40.54 months in 236887_at, $\mathrm{P}<0.05$ ). Furthermore, compared with patients with EOC with low mRNA expression levels of KIN17, patients with EOC with high expression levels of KIN17 had a poorer PFS (median, 21.13 months vs. 18.27 months in 205664_at; $\mathrm{P}<0.01$; Fig. 1H and J).

Difference in KIN17 expression between EOC andEOC-adjacent tissues. The expression levels of KIN17 were detected by western blotting in EOC and adjacent tissues. As shown in Fig. 2A and B, compared with EOC-adjacent tissues, EOC tissues exhibited higher KIN17 protein expression $(\mathrm{P}<0.05)$. 
Analysis of the association between KIN17 expression and the clinical/pathological features of patients with EOC. Using IHC staining and scoring, the expression levels of KIN17 in EOC tissues were used to divide cases into low expression (score $\leq 4 ; 24$ cases) and high expression (score $>4 ; 32$ cases) groups. Cellular yellowish or brownish staining was scored as positive. IHC staining of KIN17 in various pathologic categories of low grade or high grade is shown in Fig. 2C. The nuclear and cytoplasmic expression of KIN17 is also indicated in Fig. 2C. In the present study, the median age of the 56 patients with EOC was 52 years. All features, including age, preoperative CA125 and HE4, tumor size, lymphatic metastasis, FIGO stage, EOC type, pathological grade, and ascites volume, are summarized in Table I. Following further analysis, only lymphatic metastasis was identified to be associated with the expression levels of KIN17 $(\mathrm{P}<0.05)$. More specifically, the protein expression levels of KIN17 were higher in patients with EOC who underwent lymphatic metastasis compared with in patients with EOC without lymphatic metastasis (76.47 vs. 42.85\%; $\mathrm{P}<0.05$ ).

Analysis of risk factors for the OS of patients with EOC. After following up the 56 patients with EOC, 3- and 5-year OS rates were calculated, and all features, as well as the expression levels and location of KIN17, were evaluated (Table II). As shown in Table II and Fig. 2D, compared with those of patients with EOC with high protein expression levels of KIN17, both the 3- and 5 -year OS rates were higher in patients with EOC with low protein expression levels of KIN17 (3-year OS, 82.0\% in the EOC patients with low protein expression levels of KIN17 vs. 56.8\% in the EOC patients with high protein expression levels of KIN1; 5-year OS, 60.7 vs. 35.4\%; $\mathrm{P}<0.05$ ). This data suggested that high KIN17 expression was one of the risk factors for OS in patients with EOC. However, no association was observed between the location of KIN17 expression and OS ( $\mathrm{P}>0.05)$. Furthermore, it was identified that advanced stage (stage III+IV), type II, high grade and positive lymphatic metastasis were risk factors for the OS of patients with EOC (Table II; Fig. 2D; P<0.05).

In addition, to exclude the impact of confounding factors, Cox regression analysis was utilized to verify the independent risk factors for OS in patients with EOC. It was concluded that both advanced stage and high expression levels of KIN17 were independent risk factors for OS in patients with EOC (Table III). More precisely, the risk of death in patients with advanced-stage EOC was 6.510 times higher than that in patients who were at an early stage (stage I+II; $\mathrm{P}<0.05$ ). The risk of death in patients with EOC with high expression levels of KIN17 was 2.828 times greater than that in patients with low expression $(\mathrm{P}<0.05)$.

Association between KIN17 expression and the proliferation, as well as cisplatin sensitivity, of ovarian cancer cells. To further investigate the effects of KIN17 on EOC, shRNA-KIN17 was used to silence KIN17 expression, and a stably transfected SKOV3 cell line was generated. The transfection efficiency was examined by fluorescence microscopy (Fig. 3A), RT-qPCR (P<0.05; Fig. 3B) and western blotting $(\mathrm{P}<0.05$; Fig. $3 \mathrm{C}$ and $\mathrm{D})$. As shown in Fig. 3E, from day 4 , compared with the shRNA-Scramble group, the cell proliferation of SKOV 3 cells in the shRNA-KIN17 group was markedly lower $(\mathrm{P}<0.05)$, which implied that KIN17 knockdown could inhibit the proliferation of SKOV3 cells. Fig. 3F shows that between the concentrations of cisplatin 0.5 to $4 \mu \mathrm{g} / \mathrm{ml}$, compared with the shRNA-Scramble group, the cell proliferation of SKOV3 cells in the shRNA-KIN17 group was markedly lower $(\mathrm{P}<0.05)$, which suggested KIN17 knockdown sensitized SKOV 3 cells to cisplatin.

Migration ability of EOC cells is inhibited by downregulation of KIN17. A trans-well assay was used to detect the migration ability of EOC cells. As shown in Fig. 3G, cisplatin at $1 \mu \mathrm{g} / \mathrm{ml}$ inhibited the migration ability of SKOV3 cells. Following treatment with shRNA-KIN17, the number of cells migrating through the polycarbonate membrane was significantly decreased in the combine group (Fig. 3G), indicating that KIN17 knockdown could inhibit the migration ability of EOC cells. Therefore, the experimental data implied a notable role of KIN17 in the migration ability of EOC cells.

\section{Discussion}

Although the KIN17 protein serves an important role in human cells, it is not a highly expressed protein in all human tissues. Normally, KIN17 expression in the majority of normal tissues is low, while KIN17 upregulation has been detected in various cancer tissues (27), such as breast cancer (16), cervical cancer (17), liver cancer (18) and lung cancer (19). Compared with the normal breast cell line HS578Bst, the expression levels of KIN17 are upregulated in MDA-MB-231, SKBr-3, BT474, and MCF-7 cell lines (breast carcinoma) (16). Moreover, compared with immortalized epidermal keratinocyte line $\mathrm{HaCaT}$, the expression levels of KIN17 are upregulated in H1299, K562 (colorectal carcinoma), PC3 (prostatic carcinoma) and HeLa cell lines (cervical carcinoma) (28). These results suggest that KIN17 might be closely associated with the development of malignancies.

In the present study, the expression levels of KIN17 were demonstrated to be markedly upregulated in EOC tissues compared with EOC-adjacent tissues, borderline EOC tissues and normal ovary tissues. The present in vitro experimental results demonstrated that KIN17 silencing inhibited the proliferation of EOC cells. These results implied that the expression levels of KIN17 were associated with the oncogenesis and development of EOC. Furthermore, advanced stages (stage III+IV), type II, high grade and high KIN17 expression were identified as risk factors for OS in patients with EOC based on Kaplan-Meier univariate analysis. In addition, the study revealed that both advanced disease stage and high expression levels of KIN17 were independent risk factors based on Cox multivariate analysis. These results provided strong clinical evidence that KIN17 may have prognostic value in patients with EOC. The clinical data demonstrated that the KIN17 protein was closely associated with the OS of patients with EOC.

The combination of cytoreductive surgery and platinum-based chemotherapy is the standard treatment for patients with EOC (29). Homologous recombination is one of the important mechanisms of DNA damage repair and subsequent chemotherapeutic resistance in tumor cells (30). Therefore, exploring molecules that could regulate homologous recombination and thus prevent platinum resistance in 
Table II. Kaplan-Meier univariate analysis of OS in patients with EOC.

\begin{tabular}{|c|c|c|c|c|}
\hline Clinical features & No. of cases & 3-year OS, \% & 5-year OS, \% & P-value \\
\hline \multicolumn{5}{|l|}{ Age, years } \\
\hline$<52$ & 27 & 72.6 & 25.8 & \\
\hline$\geq 52$ & 29 & 64.2 & 52.3 & 0.851 \\
\hline \multicolumn{5}{|l|}{ FIGO stage } \\
\hline $\mathrm{I}+\mathrm{II}$ & 23 & 91.1 & 72.9 & \\
\hline III+IV & 33 & 51.6 & 24.1 & $0.001^{\mathrm{a}}$ \\
\hline \multicolumn{5}{|l|}{ Tumor size, $\mathrm{cm}$} \\
\hline$\leq 8$ & 28 & 70.4 & 55.4 & \\
\hline$>8$ & 28 & 65.5 & 36.8 & 0.636 \\
\hline \multicolumn{5}{|l|}{ EOC type } \\
\hline $\mathrm{I}$ & 26 & 84.3 & 54.5 & \\
\hline II & 30 & 52.4 & 36.9 & $0.036^{\mathrm{a}}$ \\
\hline \multicolumn{5}{|l|}{ Pathological grade } \\
\hline Low & 23 & 86.5 & 76.9 & \\
\hline High & 33 & 54.5 & 23.3 & $0.006^{\mathrm{a}}$ \\
\hline \multicolumn{5}{|c|}{ Ascites volume, ml } \\
\hline$<500$ & 31 & 73.6 & 57.2 & \\
\hline$\geq 500$ & 25 & 61.7 & 36.0 & 0.488 \\
\hline \multicolumn{5}{|c|}{ Lymph node metastasis ${ }^{\mathrm{b}}$} \\
\hline Positive & 17 & 59.6 & - & \\
\hline Negative & 28 & 81.6 & 58.7 & $0.028^{\mathrm{a}}$ \\
\hline \multicolumn{5}{|l|}{$\mathrm{CA} 125, \mathrm{U} / \mathrm{ml}$} \\
\hline$<500$ & 25 & 67.0 & 48.0 & \\
\hline$\geq 500$ & 28 & 66.2 & 52.9 & 0.794 \\
\hline \multicolumn{5}{|l|}{$\mathrm{HE} 4, \mathrm{pmol} / \mathrm{l}$} \\
\hline$<500$ & 26 & 56.1 & 48.1 & \\
\hline$\geq 500$ & 12 & 91.7 & 67.9 & 0.150 \\
\hline \multicolumn{5}{|l|}{ KIN17 expression } \\
\hline High level & 32 & 56.8 & 35.4 & \\
\hline Low level & 24 & 82.0 & 60.7 & $0.024^{\mathrm{a}}$ \\
\hline \multicolumn{5}{|c|}{ KIN17 localization } \\
\hline Cytoplasm & 18 & 62.9 & 52.4 & \\
\hline Nucleus & 26 & 63.2 & 45.1 & 0.585 \\
\hline
\end{tabular}

${ }^{a} \mathrm{P}<0.05$. EOC, epithelial ovarian cancer; FIGO, Federation International of Gynecology and Obstetrics; HE4, human epididymis protein 4; KIN17, Kin17 DNA and RNA binding protein; OS, overall survival. 'bata incomplete as some patients could not tolerate the comprehensive staging laparotomy surgery, so lymph node excision was not performed.

Table III. Multivariate Cox regression analysis of overall survival in patients with epithelial ovarian cancer.

\begin{tabular}{lclc}
\hline Prognostic factors & $\begin{array}{c}\text { Estimation of regression } \\
\text { coefficient (B) }\end{array}$ & HR (95\% CI) & P-value \\
\hline FIGO stage & 1.873 & $6.510(1.877,22.575)$ & $0.006^{\mathrm{a}}$ \\
KIN17 expression & 1.040 & $2.828(1.035,7.732)$ & $0.043^{\mathrm{a}}$ \\
\hline
\end{tabular}

${ }^{\mathrm{a}} \mathrm{P}<0.05 .95 \% \mathrm{CI}, 95 \%$ confidence interval; FIGO, Federation International of Gynecology and Obstetrics; HR, hazard ratio; KIN17, Kin17 DNA and RNA binding protein.

EOC is an important line of research. Previous studies have suggested that KIN17 could induce chemotherapy resistance in cancer cells by initiating the homologous recombination signaling pathway, resulting in the poor prognosis of patients 
A
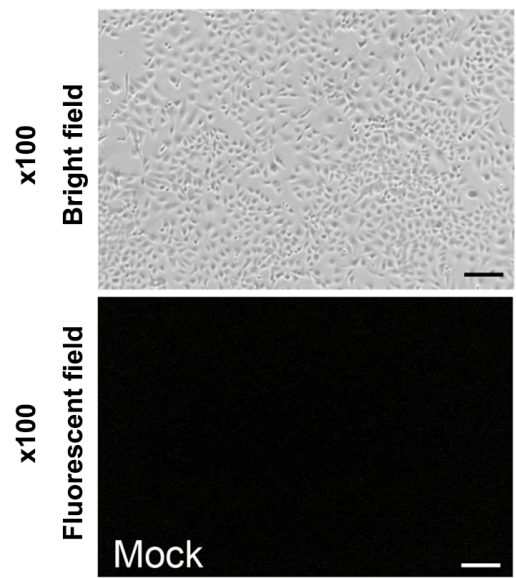
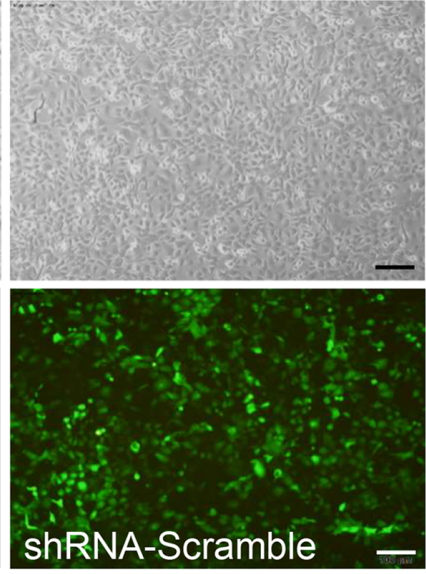
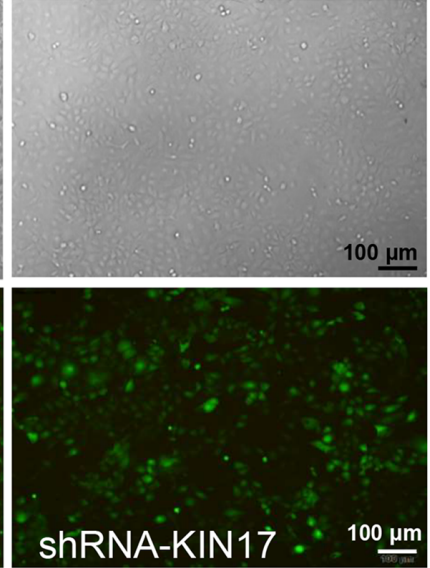

B

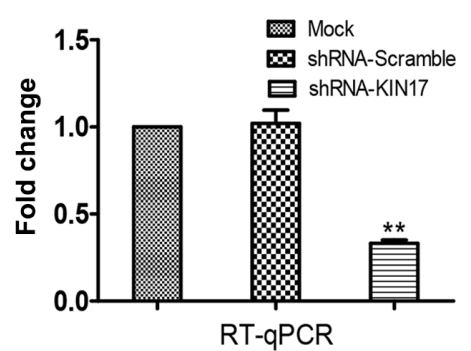

E

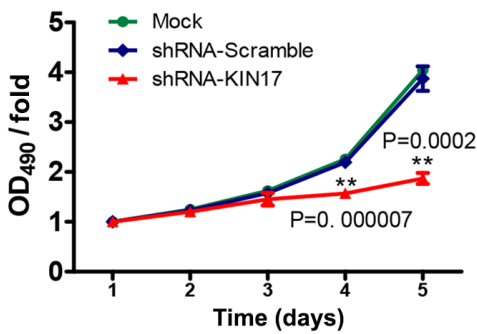

C

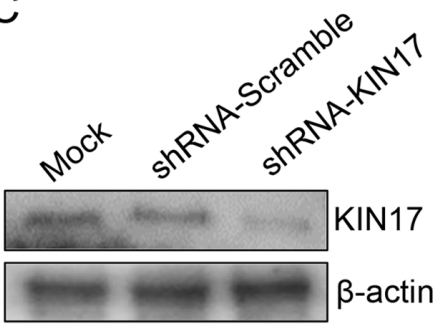

F

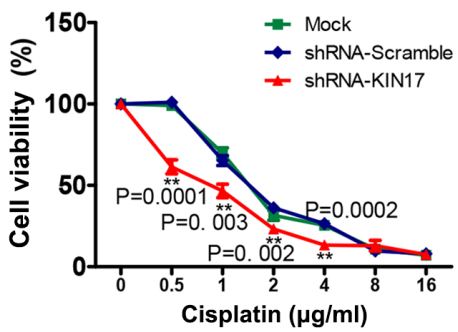

D

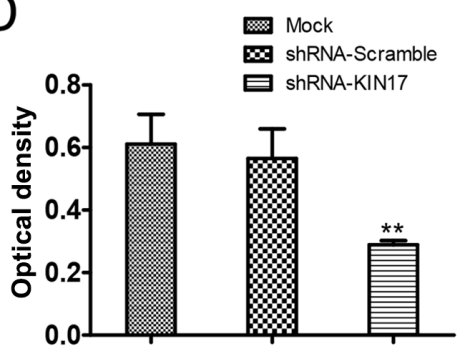

G

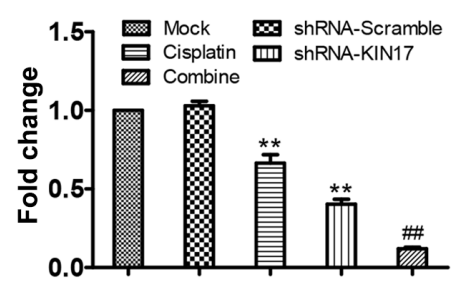

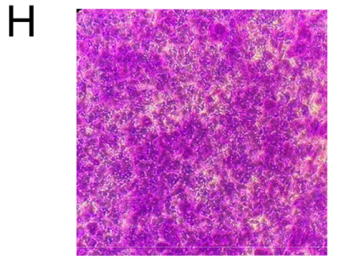

Mock

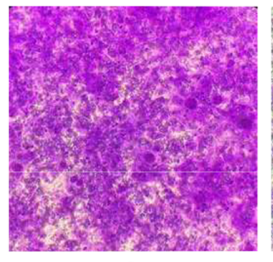

shRNA-Scramble

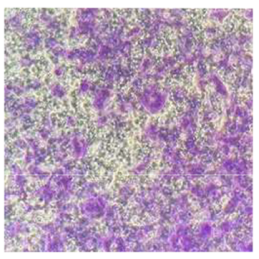

Cisplatin

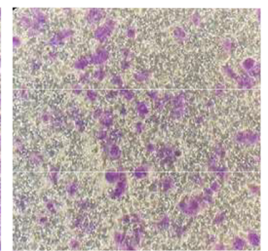

shRNA-KIN17

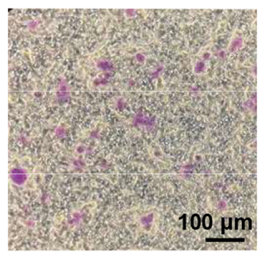

Combine

Figure 3. Association of KIN17 expression with cell proliferation, cisplatin sensitivity and migration ability of SKOV3 cells. (A) Following stable transfection with shRNA-KIN17, a fluorescence microscope was used to detect the transfection efficiency. (B) Transcription and (C) Western blot of KIN17 in SKOV3 cells were evaluated to detect the transfection efficiency. (D) Analysis of KIN17 expression levels in SKOV3 cells were evaluated to detect the transfection efficiency. (E) Proliferation rates of SKOV3 cells after transfection with shRNA were detected using a Cell Counting Kit-8 assay. (F) Sensitivity of SKOV3 cells to cisplatin after transfection with shRNA were detected using a Cell Counting Kit- 8 assay. (G) Integrated OD fold changes of the treatment groups were measured and analyzed. (H) Migration abilities of SKOV3 cells transfected with shRNA were observed using a Transwell assay (magnification, x100). ${ }^{* *} \mathrm{P}<0.01$ vs. scramble group; ${ }^{\# \#} \mathrm{P}<0.01$ vs. cisplatin group. KIN17, Kin17 DNA and RNA binding protein; OD, optical density; shRNA, short hairpin RNA.

with cancer (14,31-33). The results of the bioinformatics and clinical analyses demonstrated that patients with EOC with high KIN17 expression had a poorer prognosis as compared with the patients with low KIN17 expression, which suggested that KIN17 could be a prognostic biomarker for EOC. However, to the best of our knowledge, the mechanisms are still unclear. Bioinformatics data suggested that high mRNA expression levels of KIN17 might be associated with cisplatin resistance in EOC cell lines. Furthermore, the present in vitro analysis revealed that KIN17 knockdown could increase the sensitivity of EOC cells to cisplatin, which might be associated with the failure of the homologous recombination signaling pathway in the cells. These results imply that patients with EOC with low expression levels of KIN17 might be more sensitive to cisplatin and have an improved prognosis.

Migration of EOC cells is markedly related to the prognosis of patients (34). A previous study suggested that KIN17 might be a prognostic biomarker of non-small cell lung cancer (NSCLC), since it serves a key role in the invasion and metastasis of NSCLC cells (19). The effect of KIN17 on 
cell invasion has also been observed in cervical cancer (17). In the present study, the clinical data suggested that patients with EOC with high KIN17 expression were more likely to suffer from lymph node metastasis. Subsequent experiments indicated that KIN17 silencing resulted in inhibition of EOC cell migration in vitro. Furthermore, the anti-migration effect of cisplatin might be enhanced by KIN17 knockdown in vitro. Therefore, it was hypothesized that EOC cells in the tissues of patients with low expression levels of KIN17 may be less likely to migration, leading to an improved prognosis.

In conclusion, the present study revealed that the expression levels of KIN17 were increased in EOC. High KIN17 expression may be associated with the oncogenesis and development of EOC. Additionally, high expression levels of KIN17 were associated with lymph node metastasis, which might lead to a poor prognosis. Furthermore, KIN17 knockdown inhibited the proliferation and migration of EOC cells and sensitized EOC cells to cisplatin in vitro. Therefore, KIN17 may be an ideal candidate for therapy and a prognostic biomarker of EOC, which warrants further exploration.

\section{Acknowledgements}

Not applicable.

\section{Funding}

The present study was supported by grants from the Jilin Province Development and Reform Commission (grant no. 2014G073), the Jilin Province of Department Finance (grant no. 2019SCZT040) and the Jilin Province Science and Technology Department (grant no. 20200201589JC).

\section{Availability of data and materials}

The datasets used and/or analyzed during the current study are available from the corresponding author on reasonable request.

\section{Authors' contributions}

JC, SZ and LZ conceived and designed the experiments. YX, YP, SW, WL and HZ performed the experiments. TW, ZY and YP collected and analyzed the data. JC and SW interpreted the results and wrote the manuscript. JC, YX and SZ assessed and confirmed the authenticity of all the raw data. All authors agree to be accountable for all aspects of the research and to guarantee the accuracy and integrity of any part of the work. All authors read and approved the final manuscript.

\section{Ethics approval and consent to participate}

The present study was performed in accordance with standard guidelines and was approved by the Ethics Committee of the Second Hospital of Jilin University (approval no. 2018218; Changchun, China). All patients provided written informed consent prior to the study.

\section{Patient consent for publication}

Not applicable.

\section{Competing interests}

The authors declare that they have no competing interests.

\section{References}

1. Lheureux S, Gourley C, Vergote I and Oza AM: Epithelial ovarian cancer. Lancet 393: 1240-1253, 2019.

2. Ferlay J, Soerjomataram I, Dikshit R, Eser S, Mathers C, Rebelo M, Parkin DM, Forman D and Bray F: Cancer incidence and mortality worldwide: Sources, methods and major patterns in GLOBOCAN 2012. Int J Cancer 136: E359-E386, 2015.

3. Jayson GC, Kohn EC, Kitchener HC and Ledermann JA: Ovarian cancer. Lancet 384: 1376-1388, 2014.

4. Griffiths RW, Zee YK, Evans S, Mitchell CL, Kumaran GC, Welch RS, Jayson GC, Clamp AR and Hasan J: Outcomes after multiple lines of chemotherapy for platinum-resistant epithelial cancers of the ovary, peritoneum, and fallopian tube. Int J Gynecol Cancer 21: 58-65, 2011.

5. Boussios S, Karihtala P, Moschetta M, Karathanasi A, Sadauskaite A, Rassy E and Pavlidis N: Combined strategies with poly (ADP-Ribose) polymerase (PARP) inhibitors for the treatment of ovarian cancer: A literature review. Diagnostics (Basel) 9: 87, 2019.

6. Luvero D, Milani A and Ledermann JA: Treatment options in recurrent ovarian cancer: Latest evidence and clinical potential. Ther Adv Med Oncol 6: 229-239, 2014.

7. Dasari S and Tchounwou PB: Cisplatin in cancer therapy: Molecular mechanisms of action. Eur J Pharmacol 740: 364-378, 2014.

8. Wang QE, Milum K, Han C, Huang YW, Wani G, Thomale J and Wani AA: Differential contributory roles of nucleotide excision and homologous recombination repair for enhancing cisplatin sensitivity in human ovarian cancer cells. Mol Cancer 10: 24, 2011.

9. Borst P, Rottenberg S and Jonkers J: How do real tumors become resistant to cisplatin? Cell Cycle 7: 1353-1359, 2008.

10. Norouzi-Barough L, Sarookhani MR, Sharifi M, Moghbelinejad S, Jangjoo S and Salehi R: Molecular mechanisms of drug resistance in ovarian cancer. J Cell Physiol 233: 4546-4562, 2018.

11. Burdova K, Storchova R, Palek M and Macurek L: WIP1 promotes homologous recombination and modulates sensitivity to PARP inhibitors. Cells 8: 1258, 2019.

12. Lord CJ and Ashworth A: The DNA damage response and cancer therapy. Nature 481: 287-294, 2012.

13. Jiang QG, Xiong CF and Lv YX: Kin17 facilitates thyroid cancer cell proliferation, migration, and invasion by activating p38 MAPK signaling pathway. Mol Cell Biochem 476: 727-739, 2021.

14. The Cancer Genome Atlas Research Network: Integrated genomic analyses of ovarian carcinoma. Nature 474: 609-615, 2011.

15. Denis SFB, Miccoli L, Despras E, Frobert Y, Creminon C and Angulo JF: Ionizing radiation triggers chromatin-bound kin17 complex formation in human cells. J Biol Chem 277: 19156-19165, 2002.

16. Zeng T, Gao H, Yu P, He H, Ouyang X, Deng L and Zhang Y: Up-regulation of kin17 is essential for proliferation of breast cancer. PLoS One 6: e25343, 2011.

17. Zhang Y, Gao H, Gao X, Huang S, Wu K, Yu X, Yuan K and Zeng T: Elevated expression of Kin17 in cervical cancer and its association with cancer cell proliferation and invasion. Int J Gynecol Cancer 27: 628-633, 2017.

18. Kou WZ, Xu SL, Wang Y, Wang LW, Wang L, Chai XY and Hua QL: Expression of Kin17 promotes the proliferation of hepatocellular carcinoma cells in vitro and in vivo. Oncol Lett 8: 1190-1194, 2014.

19. Zhang Y, Huang S, Gao H, Wu K, Ouyang X, Zhu Z, Yu X and Zeng T: Upregulation of KIN17 is associated with non-small cell lung cancer invasiveness. Oncol Lett 13: 2274-2280, 2017.

20. Lu KH, Patterson AP, Wang L, Marquez RT, Atkinson EN, Baggerly KA, Ramoth LR, Rosen DG, Liu J, Hellstrom I, et al: Selection of potential markers for epithelial ovarian cancer with gene expression arrays and recursive descent partition analysis. Clin Cancer Res 10: 3291-3300, 2004.

21. Anglesio MS, Arnold JM, George J, Tinker AV, Tothill R, Waddell N, Simms L, Locandro B, Fereday S, Traficante N, et al: Mutation of ERBB2 provides a novel alternative mechanism for the ubiquitous activation of RAS-MAPK in ovarian serous low malignant potential tumors. Mol Cancer Res 6: 1678-1690, 2008. 
22. Györffy B, Surowiak P, Kiesslich O, Denkert C, Schäfer R Dietel $\mathrm{M}$ and Lage H: Gene expression profiling of 30 cancer cell lines predicts resistance towards 11 anticancer drugs at clinically achieved concentrations. Int J Cancer 118: 1699-1712, 2006.

23. Gyorffy B, Lánczky A and Szállási Z: Implementing an online tool for genome-wide validation of survival-associated biomarkers in ovarian-cancer using microarray data from 1287 patients. Endocr Relat Cancer 19: 197-208, 2012.

24. Heintz AP, Odicino F, Maisonneuve P, Quinn MA, Benedet JL, Creasman WT, Ngan HY, Pecorelli S and Beller U: Carcinoma of the ovary. FIGO 26th annual report on the results of treatment in gynecological cancer. Int J Gynaecol Obstet 95 (Suppl 1): S161-S192, 2006.

25. Kong D, Chen J, Sun X, Lin Y, Du Y, Huang D, Cheng H, He P, Yang L, Wu S, et al: GRIM-19 over-expression represses the proliferation and invasion of orthotopically implanted hepatocarcinoma tumors associated with downregulation of Stat3 signaling. Biosci Trends 13: 342-350, 2019.

26. Livak KJ and Schmittgen TD: Analysis of relative gene expression data using real-time quantitative PCR and the 2(-Delta Delta C(T)) method. Methods 25: 402-408, 2001.

27. Kannouche P, Mauffrey P, Pinon-Lataillade G, Mattei MG, Sarasin A, Daya-Grosjean L and Angulo JF: Molecular cloning and characterization of the human KIN17 cDNA encoding a component of the UVC response that is conserved among metazoans. Carcinogenesis 21: 1701-1710, 2000.
28. Despras E, Miccoli L, Créminon C, Rouillard D, Angulo JF and Biard DS: Depletion of KIN17, a human DNA replication protein, increases the radiosensitivity of RKO cells. Radiat Res 159: 748-758, 2003

29. Ovarian cancer. Nat Rev Dis Primers 2: 16062, 2016.

30. Haynes B, Saadat N, Myung B and Shekhar MP: Crosstalk between translesion synthesis, Fanconi anemia network, and homologous recombination repair pathways in interstrand DNA crosslink repair and development of chemoresistance. Mutat Res Rev Mutat Res 763: 258-266, 2015.

31. Prat J: New insights into ovarian cancer pathology. Ann Oncol 23 (Suppl 10): x111-x117, 2012.

32. Doufekas K and Olaitan A: Clinical epidemiology of epithelial ovarian cancer in the UK. Int J Womens Health 6: 537-545, 2014.

33. Chen H, Lisby M and Symington LS: RPA coordinates DNA end resection and prevents formation of DNA hairpins. Mol Cell 50: 589-600, 2013.

34. Xue Z, Zhu X and Teng Y: Long non-coding RNA CASC2 inhibits progression and predicts favorable prognosis in epithelial ovarian cancer. Mol Med Rep 18: 5173-5181, 2018.

cc) (7) $\ominus$ This work is licensed under a Creative Commons (c) Attribution-NonCommercial-NoDerivatives 4.0 International (CC BY-NC-ND 4.0) License. 